\title{
RIGHT FULLY IDEMPOTENT RINGS NEED NOT BE LEFT FULLY IDEMPOTENT
}

\author{
by R. R. ANDRUSZKIEWICZ and E. R. PUCZYŁOWSKI
}

(Received 14 September, 1993)

All rings in this paper are associative but not necessarily with an identity. The ring $R$ with an identity adjoined will be denoted by $R^{\#}$. $I<1 R$ ).

To denote that $I$ is an ideal (right ideal, left ideal) of a ring $R$ we write $I \triangleleft R\left(I<_{\mathrm{r}} R\right.$,

A ring $R$ is called right (left) fully idempotent if for every $I<_{\mathrm{r}} R\left(I<_{1} R\right), I=I^{2}$.

At the conference "Methoden der Modul und Ringtheorie" in Oberwolfach, Germany in 1993, J. Clark raised the question as to whether every right fully idempotent ring is left fully idempotent (see also [3]). A similar question was raised by S. S. Page in [5]. In this note we answer the questions in the negative.

We start with some general observations most of which are perhaps well known. We include their simple proofs for completeness.

Proposition 1. The class $\mathscr{I}$ of right (left) fully idempotent rings is a hereditary radical class.

Proof. We prove the proposition for right fully idempotent rings. It is clear that the class $\mathscr{I}$ is homomorphically closed and if a ring $R$ is the union of a chain of ideals in $\mathscr{I}$ then $R \in \mathscr{I}$.

Now we shall show that the class $\mathscr{I}$ is closed under extension. Let $I \triangleleft R$ and $I, R / I \in \mathscr{I}$. Take $H<_{\mathrm{r}} R$. Since $R / I \in \mathscr{I},((H+I) / I)^{2}=(H+I) / I$. Hence $H \subseteq H^{2}+I$. Since $I \in \mathscr{I}, H \cap I=(H \cap I)^{2} \subseteq H^{2}$. Now $H \subseteq H \cap\left(H^{2}+I\right)=H^{2}+H \cap I \subseteq H^{2}$, and so $H=H^{2}$.

It remains to prove that the class $\mathscr{I}$ is hereditary. Let $I \triangleleft R, R \in \mathscr{I}$ and $H<_{\mathrm{r}} I$. Since $R \in \mathscr{I}$ and $H R^{\#}<_{\mathrm{r}} R,\left(H R^{\#}\right)^{2}=H R^{\#}$. However $I \triangleleft R$, so $\left(H R^{\#}\right)^{2}=H R^{*} H R^{*} \subseteq H I$. Hence $H \subseteq H R^{\#} \subseteq H I \subseteq H$. Consequently $H=H R^{\#}<_{\mathrm{r}} R$ and $H^{2}=H$.

Note that in [2] K. I. Beidar proved (see also [6]) that every right (left) hereditary radical class contained in the class of idempotent rings consists of reduced von Neumann regular rings.

Proposition 2. A ring $R$ is right (left) fully idempotent if and only if for every $r \in R$, $r \in(r R)^{2}\left(r \in(R r)^{2}\right)$.

Proof. If $R$ is right fully idempotent then, for every $r \in R, r \in r R^{\#}=\left(r R^{\#}\right)^{2}=$ $r R^{\#} r R^{\#} \subseteq r R=(r R)^{2}$. Conversely if $H<_{\mathrm{r}} R$ and, for every $r \in H, r \in(r R)^{2}$ then $H \subseteq$ $(H R)^{2} \subseteq H^{2}$, so $H=H^{2}$.

Dual arguments give the characterization of left fully idempotent rings.

If $R$ is a von Neumann regular ring then, for every $r \in R$, there exists $x \in R$ such that $r=r x r$. Hence $r=r x r x r$, so $r \in(r R)^{2}$ and $r \in(R r)^{2}$. Consequently every von Neumann regular ring is right and left fully idempotent.

Note that if $a, r$ are elements of a ring $R$ such that $r=r a(r=a r)$ then $(r-a r)^{2}=0$ $\left((r-r a)^{2}=0\right)$. Hence if the ring $R$ is reduced then $r=r a$ if and only if $r=a r$.

Glasgow Math. J. 37 (1995) 155-157. 
Consequently a reduced ring is right fully idempotent if and only if it is left fully idempotent.

Proposition 2 also gives the next result.

COROLlary 1. A simple idempotent ring $R$ is right (left) fully idempotent if and only if, for every $r \in R, r \in r R(r \in R r)$. In particular every simple ring with an identity is right and left fully idempotent.

Given a ring $A$, let $r(A)=\{a \in A \mid A a=0\}$.

COROLLARY 2. If $0 \neq L<_{1} R$ and $R$ is a simple von Neuman regular ring then $L / r(L)$ is a simple right fully idempotent ring.

Proof. If $r(L) \subseteq I \triangleleft L$ and $r(L) \neq I$, then $0 \neq L I R \triangleleft R$, so $L I R=R$. However, since $R$ is von Neumann regular, $L^{2}=L$. Thus $I \supseteq L I R L=R L=L$. This shows that $L / r(L)$ is a simple ring. Now, since $R$ is von Neumann regular, for every $l \in L$, there exists $x \in R$ such that $l=l x l \in l L$. Hence by Corollary $1, L / r(L)$ is right fully idempotent.

Lemma (cf. [1]). If $L$ is a maximal left ideal of a simple non-Artinian ring $R$ with an identity then, for every non-zero $r \in R, L r \neq 0$.

Proof. Suppose $L r=0$. Then $L \subseteq \operatorname{Ker} f$, where $f: R \rightarrow R r$ is the $R$-module epimorphism defined by $f(x)=x r$. Since $L$ is maximal, $R r \cong R / L$ is a simple $R$-module. Consequently Soc $R \neq 0$. However $\operatorname{Soc} R \triangleleft R$, so $\operatorname{Soc} R=R$. Since $R$ is a ring with an identity, $R$ is a ring of finite length. Therefore $R$ is Artinian, a contradiction.

Example. Let $A$ be a simple non-Artinian von Neumann regular ring with an identity (see for instance [4, Example 8.1]), and let $L$ be a maximal left ideal of $A$. Observe that

$$
R=\left[\begin{array}{ll}
L & A \\
L & A
\end{array}\right]
$$

is a maximal left ideal of the ring $M_{2}(A)$ of $2 \times 2$-matrices over $A$. The ring $M_{2}(A)$ is non-Artinian and von Neumann regular. Hence by Lemma 1 and Corollary 2, the ring $R$ is right fully idempotent. Observe that if 1 is the identity of $A$ then

$$
\left[\begin{array}{ll}
0 & 1 \\
0 & 0
\end{array}\right] \notin\left[\begin{array}{ll}
0 & L \\
0 & L
\end{array}\right]=R\left[\begin{array}{ll}
0 & 1 \\
0 & 0
\end{array}\right]
$$

so $R$ is not left fully idempotent.

The above constructed ring is a right but not left fully idempotent simple ring without an identity. By Corollary 1 one cannot construct a simple ring with an identity of this sort. However it is not hard to modify the example to obtain a right but not left fully idempotent ring with an identity. Namely take $A$ to be a simple non-Artinian von Neumann regular algebra with an identity over a finite prime field $F$ (Example 8.1 of [4] can be applied). Then the above constructed $R$ is also an $F$-algebra. There exists an $F$-algebra $R^{*}$ with an identity such that $R \triangleleft R^{*}$ and $R^{*} / R \cong F$. Applying Proposition 1 one gets that $R^{*}$ is right but not left fully idempotent.

Acknowledgement. The work was supported by the Technical University of Białystok. 


\section{REFERENCES}

1. K. I. Beĭdar, Atoms in the "lattice" of radicals, Mat. Issled. 85 (1985), 21-31 (in Russian).

2. K. I. Beřdar, On questions of B. J. Gardner and A. D. Sands, J. Austral. Math. Soc., Ser. A 56 (1994), 314-319.

3. J. Clark, On eventually idempotent rings, Rings, modules and radicals. Proceedings of the Hobart Conference held at the University of Tasmania, Hobart, August 1987, Ed. B. J. Gardner, Pitman Research Notes in Mathematics Series 204 (Longman, 1989), 34-40.

4. K. R. Goodearl, von Neumann Regular Rings, (Pitman, London, 1979).

5. S. S. Page, Stable rings, Canad. Math. Bull. 23 (1980), 173-178.

6. E. R. Puczylowski, Some questions concerning radicals of associative rings, Theory of radicals, Colloq. Math. Soc. János Bolyai 61 (North Holland, 1993), 209-227.

INSTITUTE OF MATHEMATICS

UNIVERSITY OF WARSAW

BIAEYSTOK DIVISION

AKADEMICKA 2

15-267 BIAtYSTOK

POLAND
INSTITUTE OF MATHEMATICS UNIVERSITY OF WARSAW

BANACHA 2 02-097 WARSAW

POLAND 